\title{
A UK hospital survey to explore healthcare professional views and attitudes to patients incorrectly labelled as penicillin allergic: an antibiotic stewardship patient safety project
}

\author{
Michael Wilcock, ${ }^{1}$ Neil Powell, ${ }^{1}$ Jonathan Sandoe ${ }^{2}$
}

\begin{abstract}
- Additional material is published online only. To view please visit the journal online (http://dx.doi.org/ 10.1136/ejhpharm-2017001451).
\end{abstract}

${ }^{1}$ Pharmacy Department, Royal Cornwall Hospitals NHS Trust, Truro, UK

${ }^{2}$ Leeds Teaching Hospitals NHS Trust, Leeds, UK

\section{Correspondence to} Mr Michael Wilcock, Pharmacy Department, Royal Cornwall Hospitals NHS Trust, Truro TR1 3LQ, UK; mike.wilcock@ nhs.net

Received 15 November 2017 Revised 26 April 2018 Accepted 1 May 2018 Published Online First 24 May 2018

EAHP Statement 5: Patient Safety and Quality Assurance.

(C) European Association of Hospital Pharmacists 2019. No commercial re-use. See rights and permissions. Published by BMJ.

To cite: Wilcock $M$,

Powell N, Sandoe J.

Eur J Hosp Pharm

2019:26:329-333

\begin{abstract}
Objectives To ascertain the views, beliefs and attitudes of hospital staff to incorrect penicillin allergy records in order to determine healthcare worker motivation for the implementation of a penicillin de-labelling antibiotic stewardship intervention at the study hospital. Methods An electronic questionnaire (SurveyMonkey) was distributed to medical, nursing and pharmacy staff at a 750-bed teaching district general hospital with no specialist allergy service.

Results 193 staff responded (58\% medical, 31\% nursing and $11 \%$ pharmacy). Virtually all staff had encountered patients who believed themselves to be penicillin allergic, but felt the patient's belief to be erroneous. The potential negative consequences of an incorrectly assigned penicillin allergy label were acknowledged by the majority of respondents. In total, $188 / 190(99 \%)$ of staff thought patients having an incorrect allergy status to penicillin was a problem and required a solution. Staff reported they would feel confident using a validated evidence-based question tool to de-label patients incorrectly labelled as penicillin allergic if the process was supported by Trust management, although many still felt apprehensive about de-labelling patients for fear of patient harm through inappropriate de-labelling.

Conclusions A penicillin allergy de-labelling intervention would be well supported by healthcare workers at the study hospital, demonstrating a receptive environment for this behavioural change intervention. Further exploration of the barriers and levers to introducing an intervention is required using behavioural change methodology in order to design a successful delabelling intervention.
\end{abstract}

\section{INTRODUCTION}

The emergence of antimicrobial-resistant (AMR) bacteria is a global challenge which endangers the efficacy of antibiotics. ${ }^{1}$ One way to combat antimicrobial resistance is to identify barriers to optimal use of currently available antibiotics. Antibiotic allergies are such a barrier, particularly in penicillin-allergic patients, as penicillin-based antibiotics are often first-line treatment for common infections. A record of 'penicillin allergy' generally precludes the use of penicillins in hospitals, necessitating the utilisation of second-line agents such as quinolones or macrolides. Cephalosporin and carbapenem prescribing are also increased in penicillin-allergic patients. These second-line antibiotics are often more costly, ${ }^{2}$ can be less effective in certain clinical circumstances ${ }^{3}$ and possess a broader spectrum, increasing a patient's future risk of infection with AMR pathogens and Clostridioides difficile-associated diarrhoea. ${ }^{4}$ Compared with patients without a penicillin allergy record, those with such a penicillin allergy record are exposed to a greater number of antibiotics, experience increased length of hospital stay, increased hospital readmission rates and increased risk of dying. ${ }^{56}$

Approximately $10 \%$ of the general population have a record of penicillin allergy but, importantly, only $10 \%$ to $20 \%$ of these patients have a true allergy after formal testing. ${ }^{7-9}$ These patients are unnecessarily denied penicillins and removing incorrect records has potential to improve outcomes. An added complication is that a patient's recollection of their allergy often does not tally with their medical records. ${ }^{10} 11$

Spurious or incorrect penicillin allergy records can arise for several reasons, for example, side effects from a previous course of treatment are recorded as an 'allergy'. The prevalence of penicillin allergy records that were attributable to non-allergic side effects has been reported to be between $16 \%$ and $50 \%,{ }^{12}{ }^{13}$ with many of these patients able to safely tolerate the first-line penicillin antibiotics after appropriate investigation. Undertaking a proper history allows recategorisation in some patients from an 'allergy' record to 'non-allergic side effects', enabling safe administration of firstline penicillins. ${ }^{14}$

Guidelines suggest that antibiotic stewardship programmes should promote allergy assessment and skin testing where appropriate due to the potential impact such a programme could have on the increased utilisation of first-line agents. ${ }^{15}$ However, it is acknowledged that penicillin allergy assessment is largely unstudied as a primary antibiotic stewardship intervention, and in the UK, few hospitals provide a formal testing service as recommended in national guidelines. ${ }^{16}$

A pragmatic solution involves de-labelling, that is, removing an incorrect penicillin allergy record in patients who clearly report side effects rather than allergic reactions. ${ }^{17}$ Problems with this approach include validation of the structured questions necessary to obtain an accurate medical history from the patient; when and how to adopt such an approach in the patient's hospital journey; and how hospital staff feel about the process of explaining that the 
patient's belief or conviction of being penicillin allergic is in fact incorrect and ill founded. Though healthcare staff have been surveyed about their knowledge of penicillin allergy ${ }^{18}$ and asked about the benefits of de-labelling, ${ }^{19}$ there appears to be little reporting in the literature of how staff perceive the importance of the de-labelling process.

The aim of this study was to ascertain the views, beliefs and attitudes of hospital staff to incorrect penicillin allergy records in order to determine healthcare worker motivation, a key component of successful behavioural change, for the implementation of a penicillin de-labelling antibiotic stewardship intervention at the study hospital.

\section{METHODS}

\section{Study design and setting}

An electronic questionnaire was distributed to medical, nursing and pharmacy staff at a 750-bed teaching district general hospital with no specialist allergy service. Eligible participants had approximately 20 days to voluntarily complete the questionnaire. The hospital has a comprehensive antimicrobial stewardship programme including: implementation of the national stewardship guidelines (start smart then focus), participation in the National Health Service (NHS) England antibiotic stewardship CQUIN (Commissioning for Quality and Innovation), ${ }^{20} \mathrm{a}$ restricted antimicrobial system requiring medical microbiologist authorisation for use of restricted antibiotics for non-preapproved indications, ${ }^{21}$ daily antibiotic pharmacist antimicrobial stewardship ward rounds, and daily ward pharmacist ward rounds in which antibiotic stewardship is one of their duties and periodic medical microbiology ward rounds in haematology and intensive care. In addition, the study hospital contributes to the wider One Health antibiotic stewardship work in Cornwall. ${ }^{22}$ These components of antimicrobial stewardship mean that the hospital performs well when mapped against National Institute for Health and Care Excellence (NICE) antimicrobial stewardship guidance, ${ }^{23}$ though performs less well in relation to aspects of the NICE allergy guidance. ${ }^{7}$ This study did not require ethics approval.

\section{Questionnaire}

The questionnaire (online supplementary file) was designed using an electronic website (Survey Monkey). Most of the questions were closed multiple-choice questions (MCQs) with the exception of two open questions with opportunity for free text. Questions were developed to explore views, beliefs and attitudes towards penicillin allergy and de-labelling. The survey was piloted resulting in minor modifications. It was delivered electronically by email twice over a 2 -week period via the hospital bulletin and was also cascaded out to medical, nursing and pharmacy staff via email groups. The survey responses to closed MCQs were collated and summarised as number and percentage of responding staff using SurveyMonkey and Microsoft Excel 2013. The open questions were analysed by looking for major themes.

\section{RESULTS}

One hundred and ninety-three staff responded (58\% medical, $31 \%$ nursing and $11 \%$ pharmacy-table 1). Not all staff answered all the questions.

When asked from their knowledge and/or experience how many patients in this hospital claim to have a penicillin allergy, $60(32 \%)$ responded less than 10\%, 65 (34\%) answered more than $10 \%$ but less than $20 \%$ and $65(34 \%)$ responded more than
Table 1 Respondent's professional grade

\begin{tabular}{ll}
\hline Professional grade & $\begin{array}{l}\text { Response rate }(\mathbf{n}), \\
\mathrm{N}=193\end{array}$ \\
\hline Junior doctor & $21.2 \%(41)$ \\
\hline Senior doctor (associate specialist/consultant) & $36.3 \%(70)$ \\
Nurse/midwife band 7 or above & $7.8 \%(15)$ \\
\hline Nurse/midwife band 6 or below & $23.8 \%(46)$ \\
Pharmacist & $9.8 \%(19)$ \\
\hline Pharmacy technician & $1.0 \%(2)$ \\
\hline
\end{tabular}

$20 \%$. Virtually all staff had encountered a patient who believed themselves to be penicillin allergic, but felt the patient's belief to be erroneous-112 (58\%) frequently, 78 (40\%) occasionally, whereas $4(2 \%)$ responded they had never encountered such a patient. One hundred and five (70\%) respondents answered that they had discussed with patients the possibility that they may not be allergic, 35 (18\%) had not discussed this and 23 (12\%) were not in a position to do so. When asked why they did not discuss a mistaken penicillin allergy belief with a patient they were caring for, responses (ticking all that apply) are shown in table 2 .

There were 17 staff who answered that this is not their role to discuss with patients-13 nurses (23\% of all nurse respondents), 3 senior doctors (4\% of all senior doctor respondents) and 1 junior doctor $(2.4 \%$ of all junior doctor respondents). The 50 'other' free-text responses described main themes of: (1) risk to the patient or staff if the patient was in fact truly allergic and the staff member got it wrong (10 responses) and (2) cognition or communication difficulties with the patient (5 responses). Thirteen respondents did comment that they do discuss this with patients and medical staff, four comments emphasised lack of time to investigate the supposed allergy with the patient and any medical records and three related to the difficulty in convincing patients they are not allergic.

In total 188/190 (99\%) of staff thought patients having an incorrect allergy status to penicillin was a problem, 124/190 (65\%) staff perceived this as a problem needing an easy to implement solution and 64/190 (34\%) perceived this to be a significant problem requiring lots of time and effort devoted to resolving, with $1 \%$ perceiving it to be a minor inconvenience for healthcare staff and not worth worrying about. When asked what percentage of those patients who claim to have a penicillin allergy could safely be given a penicillin-type antibiotic without the patient coming to any serious harm, 64/189 (34\%) felt that this to be more than half of these patients.

Table 2 Reasons for not discussing a mistaken penicillin allergy belief with a patient

Response

rate $(\mathbf{n})$,

$\mathrm{N}=146$

Patients are unlikely to be convinced by my explanation that they $37.0 \%$ (54) do not have a penicillin allergy

I have come across such a patient but feel I do not have the $\quad 30.8 \%$ (45) necessary time to explain about allergies and reactions

It is not my role to discuss this with patients $\quad 11.6 \%(17)$

I have come across such a patient but feel I do not have the $\quad 9.6 \%$ (14) necessary knowledge to explain about allergies and reactions

I have come across such a patient but feel I do not have the necessary communication skills to explain about allergies and reactions 
Table 3 Statements indicated by staff to be true of patients with penicillin allergy labels when compared with those who do not have such a penicillin allergy label

\begin{tabular}{ll}
\hline Answers & $\begin{array}{l}\text { Response } \\
\text { rate (n), } \\
\mathrm{N}=194\end{array}$ \\
\hline $\begin{array}{l}\text { Have an increased risk of Clostridioides difficile-associated } \\
\text { diarrhoea. This is true. }\end{array}$ & $51.8 \%(88)$ \\
\hline $\begin{array}{l}\text { Are prescribed antibiotics that cost less. This is false. } \\
\text { Have lower readmission rates to hospital. This is false. }\end{array}$ & $2.4 \%(4)$ \\
\hline $\begin{array}{l}\text { Have higher incidence of treatment failure. This is true. } \\
\text { Have increased inpatient length of stay. This is true. }\end{array}$ & $72.4 \%(123)$ \\
\hline Have similar rates of mortality. This is false. & $59.4 \%(101)$ \\
\hline $\begin{array}{l}\text { Have increased rates of multidrug-resistant infections. This is } \\
\text { true. }\end{array}$ & $18.8 \%(32)$ \\
\hline Experience less antibiotic side effects. This is false. & $4.7 \%(8)$ \\
\hline
\end{tabular}

Regarding the implications of penicillin allergy records on antibiotic treatment choices, the majority of staff identified many potential harms that may result from using alternative antibiotic choices (table 3 ).

One hundred and two (53\%) of 194 staff felt they would be very confident using validated evidence-based questions to determine if a patient with a penicillin allergy record could be prescribed a penicillin antibiotic if such a process had Trust approval; almost half of respondents had concerns: 70/194 (36\%) would still feel a little apprehensive, 18/194 (9\%) would feel very worried about what might happen to the patient and 4/194 (2\%) respondents would not follow this process.

Thirty-two respondents provided additional free-text comments on this topic: thirteen (41\%) expressed support for a process that would assist in de-labelling patients who are not likely to be penicillin allergic; three (9\%) identified problems and difficulties with the process (one related to the patient not knowing if they have an allergy or intolerance, another identified lack of time to differentiate between allergy and intolerance, while the third bemoaned the potential role of electronic prescribing and recording in this process) and two (6\%) commented on the potential risks if a true allergy label is incorrectly removed.

\section{DISCUSSION}

Assessing all inpatients with penicillin allergy records and de-labelling those with incorrect records is not currently part of routine clinical care. To establish this practice requires new procedures and a change in the behaviour of healthcare professionals. It is increasingly recognised that behavioural change theory needs to be considered when attempting to design and implement new healthcare interventions; our work begins to explore potential barriers and facilitators to establishing de-labelling procedures.

The behaviour change wheel is a useful tool that uses the COM-B ('capability', 'opportunity', 'motivation' and 'behaviour') model to direct and prioritise elements of an intervention requiring a change in behaviour. ${ }^{24}$ This model can be further divided into physical and psychological capability, physical and social opportunity, and automatic and reflective motivation. Behaviours result from interactions involving all these components, and changing behaviour requires a change in one or more of them. In terms of psychological capability, our study found that representatives of all grades of nursing, pharmacy and medical staff were aware of the problem of incorrect penicillin allergy records and the potentially negative effect they have on patients. We found that nearly all staff had come across a patient who might have an incorrect penicillin allergy record and the majority of respondents felt this was a frequent occurrence, confirming that locally the problem is widely appreciated and this element would not be a major barrier to change.

In terms of physical capability, significant numbers of staff did not feel they had the necessary knowledge or skills to advise patients. Equipping staff for the task would be an important component of any planned intervention.

Nearly all respondents (99\%), which included senior medical staff, believed this to be a problem requiring either an easy to implement solution (65\%), or lots of time and effort devoted to resolving (34\%). Free-text comments also identified this as a long-standing issue. The survey has demonstrated that respondents are motivated to tackle the issue of incorrect penicillin allergy labels; an important finding if a de-labelling initiative is to be established. However, we elicited some preconceived ideas that might be barriers to motivation to embark on a de-labelling process with $37 \%$ (54/146) staff expressing the view that they would have difficulty in convincing patients that they are not penicillin allergic, and $11.6 \%$ (17/146) of staff believing this is not part of their role. Such concerns negatively impact the motivation of healthcare workers to tackle the issue of an incorrect penicillin allergy label and need to be addressed and overcome if a successful intervention is to be introduced. We recognise that this survey has not explored how a trust-led de-labelling initiative would ensure that any necessary changes to a patient's allergy status are conveyed to other relevant NHS staff, such as the patient's general practitioner, and community pharmacy.

In terms of the 'opportunity' component of behaviour, 70\% of respondents indicated they had already discussed the possibility with patients that they may not be penicillin allergic, demonstrating a willingness to address this issue. Those who had not discussed this possibility gave a variety of reasons, including lack of time. A previous survey of healthcare staff found that most practitioners $(197 / 274,72 \%)$ spend less than $2 \mathrm{~min}$ to assess a penicillin allergy history. ${ }^{18}$ In this context, it is the structured, detailed history taking that can distinguish a true penicillin allergy from a false positive report of allergy, and hence allow clinicians to use this important class of antibiotics when indicated. ${ }^{25}$ Ensuring that staff are supported by the environment, time and resources, including education and training, should increase the capability of the workforce to adopt the desired behaviour by impacting on physical opportunity, physical capability and psychological capability.

The majority of staff $(53 \%)$ report they would feel confident using validated evidence-based questions to determine allergy status and would be confident prescribing penicillin in a de-labelled patient if such a process had approval from the organisational management. Thirty-six per cent would feel apprehensive about such a process and 9\% very worried about patient harm with the remaining $2 \%$ not prepared to follow this process. Providing staff with validated tools to identify, as described by NICE, ${ }^{7}$ and de-label patients with an incorrect penicillin allergy label would provide physical opportunity, as well as capability through adequate training, to use the tool enabling the desired behavioural change. A validated tool would provide healthcare workers with the reassurance they need that patients will not come to harm as a result of the intervention. Personal experience of operating the validated tool and demonstrated safety of the intervention will provide positive reinforcement and motivation to perpetuate the behaviour. 
It is recognised that interventions targeting junior doctors with the aim of improving patient care are likely to be ineffective if they are expected to undertake prescribing tasks that run against the local prescribing etiquette endorsed by their seniors. ${ }^{26}$ There is a need to engage senior clinicians to ensure that the new behaviour became part of organisational culture and our study has demonstrated that a penicillin allergy de-labelling initiative would be supported by senior clinicians. This top-down and bottom-up support is postulated to be required to improve antimicrobial prescribing practice ${ }^{27}$ and demonstrates the social opportunity afforded by interpersonal influences, social cues and cultural norms ${ }^{24}$ that influence how staff perceive current behaviour around managing patients with incorrect penicillin allergy labels and how this might change.

There is a need to educate staff about the potential patient benefits, including reduced treatment failure, length of stay and readmission, of using penicillin antibiotics over second-line agents to motivate staff. There is also a clear need to convince staff of the safety of such an intervention. Providing education that promotes a wider understanding of the negative consequences of retaining an incorrect penicillin allergy label would achieve reflective motivation in the workforce.

Though both the consequences of having an incorrect penicillin allergy label and possible approaches to de-labelling have been described both in a secondary and primary care setting, ${ }^{28}$ as far as we are aware this is the first study asking hospital staff for their views on this 'malady' or problem. ${ }^{29}$ Limitations of this survey include being based solely in one acute hospital setting so results are not necessarily generalisable. It was not possible to calculate the response rate as we do not know how many staff saw the survey link and chose not to respond. As with all voluntary surveys, there is a potential for selection bias because those interested in the topic of antibiotic allergy may be more likely to respond. We acknowledge that respondents might have given false answers aiming to fulfil certain expectations, though to mitigate this response bias, questionnaires were anonymised. In addition, the chosen survey items have not been validated though they were based on the published literature, and our survey did not delve into staff views and knowledge on the various forms and severity of allergies and hypersensitivity reactions.

\section{CONCLUSIONS}

We have identified a number of areas using behavioural change theory, which need to be targeted in the design of a de-labelling intervention. Respondents perceived having an incorrect penicillin allergy label to be a problem requiring a solution, demonstrating motivation to tackle the issue of incorrect penicillin allergy labels. However, we also identified potential barriers to healthcare motivation to enacting the desired behaviour, for example, time. Opportunity to enact the behaviour is partially met with social opportunity evident and already in place, but deficiencies in physical opportunity are evident. Capability was briefly touched on with evidence on staff capability to enact the behaviour, but this is not widespread and a requirement to explore this further is necessary. In conjunction with staff focus groups we will delve further into how best to plan and deliver an intervention to develop a de-labelling initiative for patients reporting an obvious non-severe side effect to penicillin rather than an allergic reaction, with exploration of the nine intervention functions targeting the COM-B components of the behaviour change wheel and the wider seven policies that facilitate or enable these interventions.
What this paper adds

What is already known on this subject

- Antimicrobial resistance is a global problem.

- Reported penicillin allergy rarely reflects penicillin intolerance.

- Antimicrobial stewardship programmes should consider the use of structured clinical history taking to exclude erroneous penicillin allergy label.

What this study adds

- Hospital staff (doctors, nurses, pharmacy team) recognise the negative consequences of an incorrectly assigned penicillin allergy label.

- A suitably designed penicillin allergy de-labelling intervention would be well supported by hospital staff.

Funding The authors have not declared a specific grant for this research from any funding agency in the public, commercial or not-for-profit sectors.

Competing interests None declared.

Provenance and peer review Not commissioned; externally peer reviewed.

\section{REFERENCES}

1 World Health Organisation. Antimicrobial resistance: global report on surveillance. WHO, Switzerland 2014

2 Li M, Krishna MT, Razaq S, et al. A real-time prospective evaluation of clinical pharmaco-economic impact of diagnostic label of 'penicillin allergy' in a UK teaching hospital. J Clin Pathol 2014:67:1088-92.

$3 \mathrm{Kim} \mathrm{SH}, \mathrm{Kim} \mathrm{KH}$, Kim HB, et al. Outcome of vancomycin treatment in patients with methicillin-susceptible Staphylococcus aureus bacteremia. Antimicrob Agents Chemother 2008;52:192-7.

4 Blumenthal KG, Shenoy ES, Varughese CA, et al. Impact of a clinical guideline for prescribing antibiotics to inpatients reporting penicillin or cephalosporin allergy. Ann Allergy Asthma Immunol 2015;115:294-300.

5 Charneski L, Deshpande G, Smith SW. Impact of an antimicrobial allergy label in the medical record on clinical outcomes in hospitalized patients. Pharmacotherapy 2011:31:742-7.

6 Trubiano JA, Chen C, Cheng AC, et al. Antimicrobial allergy 'labels' drive inappropriate antimicrobial prescribing: lessons for stewardship. J Antimicrob Chemother 2016:71:1715-22.

7 Clinical Guideline Centre. Drug allergy: diagnosis and management of drug allergy in adults, children and young people. UK: Commissioned by the National Institute for Health and Care Excellence, 2014.

8 Mota I, Gaspar Â, Chambel M, et al. Hypersensitivity to beta-lactam antibiotics: a three-year study. Eur Ann Allergy Clin Immunol 2016;48:212-9.

9 Salkind AR, Cuddy PG, Foxworth JW. The rational clinical examination. Is this patient allergic to penicillin? An evidence-based analysis of the likelihood of penicillin allergy. JAMA 2001;285:2498-505.

10 Wyatt JP. Patients' knowledge about their drug allergies. J Accid Emerg Med 1996;13:114-5.

11 MacPherson RD, Willcox C, Chow C, et al. Anaesthetist's responses to patients' selfreported drug allergies. Br J Anaesth 2006;97:634-9.

12 Trubiano JA, Cairns KA, Evans JA, et al. The prevalence and impact of antimicrobial allergies and adverse drug reactions at an Australian tertiary centre. BMC Infect Dis 2015; 15:572.

13 Preston SL, Briceland LL, Lesar TS. Accuracy of penicillin allergy reporting. Am J Hosp Pharm 1994:51:79-84.

14 Krishna MT, Huissoon AP, Li M, et al. Enhancing antibiotic stewardship by tackling "spurious" penicillin allergy. Clin Exp Allergy 2017;47:1362-73.

15 Barlam TF, Cosgrove SE, Abbo LM, et al. Implementing an antibiotic stewardship program: guidelines by the infectious diseases Society of America and the Society for Healthcare Epidemiology of America. Clin Infect Dis 2016;62:e51-e77.

16 Mirakian R, Leech SC, Krishna MT, et al. Management of allergy to penicillins and other beta-lactams. Clin Exp Allergy 2015:45:300-27.

17 Sigona NS, Steele JM, Miller CD. Impact of a pharmacist-driven beta-lactam allergy interview on inpatient antimicrobial therapy: A pilot project. J Am Pharm Assoc 2016;56:665-9.

18 Staicu ML, Soni D, Conn KM, et al. A survey of inpatient practitioner knowledge of penicillin allergy at 2 community teaching hospitals. Ann Allergy Asthma Immunol 2017;119:42-7. 
19 Trubiano JA, Beekmann SE, Worth LJ, et al. Improving antimicrobial stewardship by antibiotic allergy delabeling: evaluation of knowledge, attitude, and practices throughout the Emerging Infections Network. Open Forum Infect Dis 2016:3:ofw153.

20 Commissioning for Quality and Innovation (CQUIN). Guidance for 2017-2019. 2016. https://www.england.nhs.uk/nhs-standard-contract/cquin/cquin-17-19/

21 Powell N, Franklin BD, Jacklin A, et al. Omitted doses as an unintended consequence of a hospital restricted antibacterial system: a retrospective observational study. J Antimicrob Chemother 2015;70:dkv264-83.

22 Powell N, Davidson I, Yelling P, et al. Developing a local antimicrobial resistance action plan: the Cornwall One Health Antimicrobial Resistance Group. J Antimicrob Chemother 2017;72:2661-5.

23 Clinical Guideline Centre. Antimicrobial stewardship: systems and processes for effective antimicrobial medicine use. 2015. https://www.nice.org.uk/guidance/ ng 15
24 Michie S, van Stralen MM, West R. The behaviour change wheel: a new method for characterising and designing behaviour change interventions. Implement Sci 2011;6:42.

25 Khasawneh FA, Slaton MA, Katzen SL, et al. The prevalence and reliability of self-reported penicillin allergy in a community hospital. Int J Gen Med 2013;6:905-9.

26 Charani E, Castro-Sanchez E, Sevdalis N, et al. Understanding the determinants of antimicrobial prescribing within hospitals: the role of "prescribing etiquette". Clin Infect Dis 2013;57:188-96.

27 Papoutsi C, Mattick K, Pearson M, et al. Social and professional influences on antimicrobial prescribing for doctors-in-training: a realist review. J Antimicrob Chemother 2017;72:2418-30.

28 Sundquist BK, Bowen BJ, Otabor U, et al. Proactive penicillin allergy testing in primary care patients labeled as allergic: outcomes and barriers. Postgrad Med 2017:129:915-20.

29 Lang DM. The malady of penicillin allergy. Ann Allergy Asthma Immunol 2016;116:269-70. 